\title{
Greater pre-stimulus effective connectivity from the left inferior frontal area to other areas is associated with better phonological decoding in dyslexic readers
}

\author{
Richard E. Frye ${ }^{1 *}$, Meng-Hung Wư ${ }^{2}$, Jacqueline Liederman ${ }^{3}$ and Janet McGraw Fisher ${ }^{4}$ \\ Department of Pediatrics, Division of Child and Adolescent Neurology and the Children's Learning Institute, University of Texas Health Science Center, Houston, TX, \\ USA \\ 2 Department of Computer Science, University of Houston, Houston, TX, USA \\ ${ }^{3}$ Department of Psychology, Boston University, Boston, MA, USA \\ ${ }^{4}$ Department of Psychology, State University of New York Westchester County, Westchester, NY, USA
}

Edited by:

Barry Horwitz, National Institutes of Health, USA

\section{Reviewed by:}

Ben Xu, National Institutes of Health, USA

Jason Smith, National Institutes of Health, USA

\section{${ }^{*}$ Correspondence:}

Richard E. Frye, Department of

Pediatrics, University of Texas Health

Science Center, 7000 Fannin-UCT

2478, Houston, TX 77030, USA

e-mail: richard.e.frye@uth.tmc.edu
Functional neuroimaging studies suggest that neural networks that subserve reading are organized differently in dyslexic readers (DRs) and typical readers (TRs), yet the hierarchical structure of these networks has not been well studied. We used Granger causality to examine the effective connectivity of the preparatory network that occurs prior to viewing a non-word stimulus that requires phonological decoding in 7 DRs and 10 TRs who were young adults. The neuromagnetic activity that occurred $500 \mathrm{~ms}$ prior to each rhyme trial was analyzed from sensors overlying the left and right inferior frontal areas (IFA), temporoparietal areas, and ventral occipital-temporal areas within the low, medium, and high beta and gamma sub-bands. A mixedmodel analysis determined whether connectivity to or from the left and right IFAs differed across connectivity direction (into vs. out of the IFAs), brain areas, reading group, and/or performance. Results indicated that greater connectivity in the low beta sub-band from the left IFA to other cortical areas was significantly related to better non-word rhyme discrimination in DRs but not TRs. This suggests that the left IFA is an important cortical area involved in compensating for poor phonological function in DRs. We suggest that the left IFA activates a wider-than usual network prior to each trial in the service of supporting otherwise effortful phonological decoding in DRs. The fact that the left IFA provides top-down activation to both posterior left hemispheres areas used byTRs for phonological decoding and homologous right hemisphere areas is discussed. In contrast, within the high gamma sub-band, betterperformance was associated with decreased connectivity between the left IFA and other brain areas, in both reading groups. Overly strong gamma connectivity during the pre-stimulus period may interfere with subsequent transient activation and deactivation of sub-networks once the non-word appears.

Keywords: Granger causality, effective connectivity, magnetoencephalography, reading, dyslexia, top-down, compensatory mechanisms

\section{INTRODUCTION}

Developmental dyslexia is the most common learning disorder worldwide, affecting both children and adults with a prevalence ranging up to $17.5 \%$ (Shaywitz, 1998). Dyslexia is a lifelong disorder with a wide variability in prognosis regardless of the quality of remediation. Reading disability may be represented on a continuum of severity with multiple genetic and environmental risk factors interacting to result in the phenotype known as dyslexia (Pennington and Lefly, 2001; Snowling, 2008). Individuals with developmental dyslexia have atypical patterns of cortical folding and migrational anomalies, both of which are consistent with a prenatal origin of dyslexia (Galaburda et al., 1985; Kaufmann and Galaburda, 1989; Humphreys et al., 1990; Frye et al., 2010a). Many neuroimaging studies have compared patterns of functional activation between dyslexic readers (DRs) and typical readers (TRs). Three regions of the brain that are often atypically activated in DRs as compared to TRs, include the inferior frontal areas (IFAs), the temporoparietal areas (TPAs) and the ventral occipital-temporal areas (VOTAs; Pugh et al., 2000a). This study will focus on the IFAs and its connection to these other two brain areas.

\section{FUNCTIONAL ACTIVATION OF THE INFERIOR FRONTAL AREA IN DYSLEXIC READERS}

The IFA has been reported to have abnormal activation in DRs as compared to TRs in several studies. However, differences in IFA activation between DRs and TRs during phonological word decoding tasks are not consistent across studies. Functional magnetic resonance imaging (fMRI) studies have reported over-activation of the left IFA (Hoeft et al., 2007; MacSweeney et al., 2009), the right IFA (Shaywitz et al., 2003), or neither IFA (Eden et al., 2004; Hoeft et al., 2006; Richards et al., 2007; MacSweeney et al., 2009) in DRs as compare to TRs during phonological word decoding tasks. Other fMRI studies have found underactivation of the IFA in DRs as compared to TRs during phonological word decoding tasks (Aylward et al., 2003; Cao et al., 2006). In fact, two meta-analyses 
of fMRI studies have not verified a consistent increase or decrease in activation of either the left or right IFAs (Maisog et al., 2008; Richlan et al., 2009).

In contrast to fMRI studies, magnetoencephalography (MEG) studies have suggested that it is not the amount of activation per se that is different between DRs and TRs, but the timing of the activation. For example, the onset of TPA activity preceded the onset of IFA activity for TRs while the onset of activity for the IFA and TPA were not different for DRs before an intervention (Simos et al., 2007).

The inconsistent findings for IFA activation may be related to variation in the characteristics of the participants, in particular, the level of remediation. Indeed, it has been repeatedly reported in fMRI studies that IFA activity increases following remediation (Richards et al., 2002; Temple et al., 2003; Eden et al., 2004). This could suggest that IFA activity has a key role in enabling better phonological function in DRs. In addition, fMRI studies have shown that IFA activity increases from childhood to adulthood for DRs but not TRs, suggesting that the maturation of the IFA occurs along a different trajectory in DRs and TRs (Brunswick et al., 1999; Shaywitz et al., 2007). It is noteworthy that the developmental increase in IFA activity from childhood through adolescence into adulthood for DRs corresponds to the course of phonological skill development for DRs that succeed in developing adequate phonological decoding skills (Miller-Shaul, 2005; Svensson and Jacobson, 2006). Thus, improvement in phonological across this time period might be related to IFA maturation.

Timing of the onset of IFA activity may also be related to remediation. In DRs, the onset of activity for the IFA and TPA were not different before remediation. After remediation TPA activity preceded IFA activity in DRs the way it usually does in TRs (Simos et al., 2006, 2007). However, the number of dipoles, the MEG measure of functional activation, in the IFA did not change after remediation for those participants that responded to the remediation therapy (Simos et al., 2006, 2007). These studies suggest that the IFA plays an integral role in phonological function, especially in the improvement of phonological function over time and with remediation, in DRs. However, there also appear to be discrepancies in functional activation between fMRI and MEG studies.

The involvement of the IFA in DRs may also be important since it subserves several aspects of executive function including inhibition and switching (Kenner et al., 2010), analogical reasoning (Hampshire et al., 2010), and updating (Tamnes et al., 2010). This is of special interest because several lines of evidence point to problems with executive function in DRs. For example, deficits in working memory (Willcutt et al., 2001; Gioia et al., 2002), planning and organization (Gioia et al., 2002), set shifting and organization (Narhi et al., 1997), inhibition (Willcutt et al., 2005), sequencing (Brosnan et al., 2002), and problem solving (Lazar and Frank, 1998) have been documented in DRs. In fact, dysfunction of the executive attentional system has recently been implicated in relation to reading ability in DRs (Shaywitz and Shaywitz, 2008). Interestingly, the IFA may have an executive role specific to language as it has been shown to be involved in regulating language networks and word learning (Pugh et al., 2000a; Aron and Poldrack, 2005). Moreover, recent studies have shown that top-down regulation of the language system from the IFA develops during childhood in TRs (Bitan et al., 2009).

\section{FUNCTIONAL CONNECTIVITY IN NORMAL AND DYSLEXIC READERS}

As opposed to just studying regions of activation and deactivation, functional connectivity has been used to illuminate how subsystems interact to enable reading. One landmark study used positron emission tomography (PET) during single word reading of exception words or pseudowords. Correlational analyses were computed within task, between regions, and across subjects (Horwitz et al., 1998). Adult DRs as compared to TRs had weaker connectivity between the left fusiform gyrus and the left angular gyrus (Horwitz et al., 1998). This finding was similar to that reported by Pugh et al. (2000b) who used fMRI to determine functional connectivity between cortical areas. Pugh et al. (2000b) concluded that adult DRs as compared to TRs had weaker connectivity in the left hemisphere between the extrastriate cortex and left angular gyrus. In the right hemisphere, however, DRs as compared to TRs had stronger connectivity between these structures. Recently Koyama et al. (2010) examined fMRI functional connectivity between key brain regions consistently implicated in reading during the resting state. Conjunction analysis identified the posterior part of the left IFA and the posterior part of the left middle temporal gyrus as loci of functional interactions with the majority of the other cortical regions involved in reading.

Newer effective connectivity techniques measure causal connectivity. Structural equation modeling and dynamic causal modeling are the two effective connectivity techniques that have been applied to reading. These techniques require the experimenter to set up a restricted number of causal models. Free parameters are estimated and then the model's fit to the data is assessed. Such effective connectivity techniques have been used in limited studies on reading in TRs (Bitan et al., 2009; Levy et al., 2009) and DRs (Cao et al., 2008; Quaglino et al., 2008). These studies have provided insight into the relationship among key areas involved in reading for DRs. A recent study using structural equation modeling applied to fMRI data obtained during a pseudoword reading task found that causal connectivity between the left supramarginal cortex and the left IFA was absent in DRs but present in TRs matched for age or reading level. In contrast, in the same study, causal connectivity between the left supramarginal cortex and the left VOTA was intact for DRs and both groups of TRs (Quaglino et al., 2008). These data imply a specific lack of connectivity between the left IFA and left TPA in DRs. Similarly, using fMRI and dynamic causal modeling, other researchers showed that the topdown influence from the left IFA to the left TPA found in TRs was absent in DRs (Cao et al., 2008). These studies provide support for the idea that interactions with IFA and posterior brain regions are abnormal in DRs. However, these model-driven techniques restrict the number of possible causal hypotheses. Consequently, some studies on reading have only evaluated one direction of coupling (e.g., feedforward; Levy et al., 2009) or analyzed only the left hemisphere (Cao et al., 2008; Quaglino et al., 2008; Bitan et al., 2009).

\section{USING GRANGER CAUSALITY TO MEASURE EFFECTIVE CONNECTIVITY}

In contrast to these model-driven techniques, Granger causality (GC) is a data-driven technique that empirically calculates the direction and strength of connectivity with minimal assumptions about the structure of the neural network. Unlike previous studies using effective connectivity techniques to study reading which have used fMRI and model-driven techniques, the current study uses GC to analyze MEG data. MEG has more than 100 times the temporal resolution of fMRI, thus providing the ability to resolve brain connectivity at 
multiple brain frequencies. However, the higher temporal resolution also allows connectivity to potentially change within the sampling window selected for analysis, thereby potentially resulting in a nonstationary signal. Using a short window can mitigate this issue, but guidelines for choosing the window size have not been investigated in MEG (Ding et al., 2000; Frye and Wu, in press). For this reason, we analyzed effective connectivity before the onset of the experimental trial, just before presentation of the stimulus. During this pre-stimulus period, brain activity reflects a relatively static preparatory state (Liang et al., 2002), allowing the assumption of stationarity with respect to brain connectivity (and the first and second moment of the signal).

\section{HYPOTHESIZED COMPENSATORY ROLE OF GREATER TOP-DOWN INFLUENCE OF THE LEFT INFERIOR FRONTAL AREA IN DYSLEXIC READERS}

One characteristic that is pervasive among DRs is a lack of automaticity in the phonological decoding systems. This has led some authors to describe DRs as having "effortful" word processing and suggest that the increased IFA activation in DRs represents this increased effort (Shaywitz and Shaywitz, 2005). However, despite this hypothesis, there is little evidence to specifically link the IFA to increased effort. Here we hypothesis that, indeed, the IFA is involved in compensating for this lack of automaticity by activating the language system though top-down influence. The optimal time to activate the language network would be prior to the onset of the stimulus, during the pre-stimulus periods.

Our main hypothesis is that the left IFA will demonstrate increased top-down influence on the posterior language brain areas (i.e., left TPA and left VOTA) for DRs but not TRs (since engagement of the language network is automatic in TRs). Moreover we predict that a greater degree of top-down activation from left IFA to these posterior areas will be associated with better phonological decoding only in DRs.

To test this hypothesis, MEG data were extracted from each trial during the period just before the first non-word was presented on the display. We used GC to measure effective connectivity between key regions implicated in the brain networks responsible for phonological word decoding and compared this connectivity between TRs and DRs. We also tested whether task performance correlated with effective connectivity during the pre-stimulus period.

\section{EXAMINING FREOUENCY BANDS TO FURTHER UNDERSTAND THE ROLE OF THE INFERIOR FRONTAL AREA}

In MEG, electroencephalogram (EEG), and intracranial studies, activity within the beta and gamma frequency sub-bands have been linked to phonological and orthographic processes required for reading (Duncan Milne et al., 2003; Mainy et al., 2008; Matsumoto and Iidaka, 2008; Cornelissen et al., 2009; Trebuchon-Da Fonseca et al., 2009; Penolazzi et al., 2010) and beta and gamma sub-bands have been reported to be different in TRs and DRs (Ortiz et al., 1992; Ackerman et al., 1994; Klimesch et al., 2001). Since beta band activity has been linked to large-scale integration of brain activity such as long range synchronization of the frontal, parietal, temporal, and occipital areas (Gross et al., 2004, 2006) and multimodal integration between cortical lobes (von Stein et al., 1999), it is hypothesized that activity within the beta frequency band will be related to large-scale inter-regional integration such as top-down control of frontal areas on posterior brain areas. In contrast to beta band activity, gamma band activity has been linked to local feature integration (Fries, 2007) and the transient coupling and uncoupling of local neural networks (Lachaux et al., 2008). Thus, we hypothesize that the gamma band activity will not be related to inter-regional integration signals like the beta-band activity.

\section{MATERIALS AND METHODS PARTICIPANTS}

We examined 10 TRs and 7 DRs native English speakers between the ages of 18 and 45 years, with normal or corrected vision, normal hearing, and no history of severe psychiatric or neurological illnesses or attention defects. DRs reported a childhood diagnosis of dyslexia and were either referred from the Office of Disability Services at Boston University or recruited from Curry College in Milton, MA, USA.

Reading performance composite was calculated by averaging the percentile ranks of reading rate and comprehension of the NelsonDenny Reading Test. DRs scored below and TRs scored above the 25 th percentile. All participants scored greater than or equal to 80 on the Wechsler Adult Intelligence Scale as estimated from vocabulary and block design subtests (Wechsler, 1997) and subtest scores were equivalent for DRs and TRs (Table 1). Right-handedness was confirmed by a score greater than 50 on the Edinburgh Handedness Inventory (Dragovic, 2004). Written informed consent was obtained in accordance with our Institutional Review Board regulations. Participants underwent an MEG and MRI scan as described below and received $\$ 20$ per hour.

\section{NON-WORD RHYME TASK}

Since equating difficulty across reading groups can be a confounding factor, a non-word rhyming task that could be manipulated to provide equivalent performance across reading groups was developed (McGraw Fisher et al., in press). The visual stimuli were projected by a Panasonic DLP projector (Model No. PT-D7500U) through an aperture in the chamber onto the back of a non-magnetic screen located $1.5 \mathrm{~m}$ in front of the participant. Non-word target items were presented for $400 \mathrm{~ms}$ each, and then the non-word test item appeared for $400 \mathrm{~ms}$. The total time from trial onset to the onset of the test item remained constant at $1650 \mathrm{~ms}$. Depending on the block, one, two, or three target items were presented sequentially before the test item. The participant's task was to indicate if any of the target non-word(s) rhymed with the test non-word. A keypad press with the right index or middle finger indicated a rhyme or non-rhyme, respectively. The inter-trial interval was $2000 \mathrm{~ms}$. Each testing block consisted of 60 randomly presented novel trials. TRs completed six blocks: four with one target item, one with two target items, and one with three target items. DRs completed five blocks:

Table 1 | Participant characteristics [mean (standard deviation)].

\begin{tabular}{lll}
\hline Characteristic & Typical readers & Dyslexic reade \\
\hline Age & $21.9(3.1)$ & $25.0(6.3)$ \\
Male:female & $5: 5$ & $3: 4$ \\
Handedness & $77.8(17.2)$ & $87.1(11.1)$ \\
Nelson-Denny rate & $35 \%(21 \%)$ & $6 \%(6 \%)$ \\
Nelson-Denny comprehension & $65 \%(32 \%)$ & $12 \%(13 \%)$ \\
Nelson-Denny average & $50 \%(21 \%)$ & $11 \%(8 \%)$ \\
Vocabulary subtest & $12.6(2.6)$ & $13.6(3.4)$ \\
Block design subtest & $12.7(1.6)$ & $11.4(2.0)$
\end{tabular}


four with one target item and one with two target items. The block with three target items was not given to the DRs since they were found in preliminary studies to perform at about chance with such a difficult phonological task. In addition, this experimental design was found to provide equivalent performance across these two reading groups (McGraw Fisher et al., in press).

\section{PERFORMANCE MEASUREMENTS}

A signal detection paradigm was used to obtain a measurement of performance without response bias. Rhyme trials were considered signal + noise trials while non-rhyme trials were considered noise trials. Sensitivity ( $d$-prime) was calculated from the hit and false alarm rates assuming an equal variance model [i.e., $z$ (hit rate) $-z$ (false alarm rate)].

\section{MAGNETIC RESONANCE IMAGING}

After the MEG session, a high-resolution, 3D, T1-weighted structural MRI of the brain was acquired. Using FreeSurfer software, the MRI images were segmented and the cortical surface was reconstructed (Dale et al., 1999; Fischl et al., 1999). These images were used to ensure that the MEG sensors selected were located above the true regions of interest.

\section{MAGNETOENCEPHALOGRAPHY ACOUISITION Participant preparation}

Four head position indicator coils were placed on both sides of the forehead and behind the ears. These coils were used to determine the relative position of the head while in the scanner. The coils' positions were measured using a low-intensity magnetic field generated by each coil at the start of each run. The positions of the coils, the nasion, and auricular points were recorded with a Polhemus Fastrack (Colchester, VT, USA) 3-D digitizer (Hämäläinen et al., 1993) and about 70 points on the scalp were marked with the digitizer. Electro-oculography (EOG) electrodes were placed at each temple and above and below the left eye, with the ground on the left lower cheek. Vertical and horizontal EOG was recorded to detect blinks and large eye movements.

\section{MEG recording}

Magnetoencephalography recordings were performed at the Massachusetts General Hospital Athinoula A. Martinos Center for Biomedical Imaging using a whole-head VectorView ${ }^{\mathrm{TM}}$ system (Elekta Neuromag Oy, Finland) inside a high performance magnetically shielded room (Imedco AG, Switzerland; Cohen et al., 2002). The device has 306 SQUID (superconducting quantum interference device) sensors arranged in 102 locations within a helmet-shaped array. Each location contained a longitudinal and latitudinal planar gradiometers and a magnetometer. Signals were filtered at $0.1-172 \mathrm{~Hz}$ and sampled at $601 \mathrm{~Hz}$.

\section{MEG data processing}

Blinking and other artifacts were excluded by removing epochs with EOG amplitudes exceeding $150 \mu \mathrm{V}$ or gradiometer signals exceeding $3000 \mathrm{fT} / \mathrm{cm}$. Typically, one or two MEG channels were excluded for each participant due to artifacts. To examine the preparatory state activity, the neural activity was extracted from 500 to $0 \mathrm{~ms}$ before the onset of the first stimulus from each trial. Approximately 300 trials were extracted for each participant. To reduce the number of channels, the signal amplitude at each location was derived from the longitudinal and latitudinal planar gradiometers as given in Eq. 1 .

Signal amplitude

$$
=\sqrt{\text { longitudinal amplitude }^{2}+\text { latitudinal amplitude }}{ }^{2}
$$

Signals were filtered into low $(12-14 \mathrm{~Hz})$, medium $(15-19 \mathrm{~Hz})$, and high $(20-29 \mathrm{~Hz})$ beta and low $(30-59 \mathrm{~Hz})$, medium $(60-89 \mathrm{~Hz})$, and high (90-120 Hz) gamma sub-bands using low-order bidirectional Butterworth filters to prevent frequency and phase distortion. The signal was down-sampled by a factor of 2 .

\section{Region of interest selection}

Data was selected from 24 sensor locations overlying the right and left IFA, TPA, and VOTA. A viewer depicting the exact position of the selected sensors over the 3D model for each participant was used to ensure that the position of the sensors corresponded to the regions of interest for each participant. The average Talairach coordinates of the cortex underlying the center of the groups of sensors for each region of interest are as follows: left IFA -54.2, 22.4, 1.71; left TPA $-62.5,-51.1,30.4$; left VOTA -42.8, -62.4,-13.2; right IFA 57.7, 26.7, 7.1; right TPA 58.0, -54.4, 35.8; right VOTA 39.2, -55.1, -14.4.

\section{GRANGER CAUSALITY ANALYSIS}

The interaction between multiple brain regions were processed using an implementation of GC we recently developed - Dynamic Autoregressive Neuromagnetic Causal Imaging (DANCI). DANCI uses least-squares linear regression (LSLR) to model the interactions between a large number of MEG sensors or sources (Frye and $\mathrm{Wu}$, in press). To calculate GC, a system of autoregressive (AR) models was constructed to represent the mutual influence of $S$ sensors on one another. The MEG signal from a set of sensors [1...S], where $S=24$, with time points $[1 \ldots T]$, where $T=20$ (see below), is given in time series $A=\left[a_{s}(t): s=1 \ldots S, t=1 \ldots T\right]$. A system of AR models of order P (see Eq. 2) was used to model the time series. The model order determines the number of coefficients that are used to model each sensor-sensor interaction.

$$
\begin{aligned}
a_{1}(t)= & \sum_{j=1}^{p} c_{1,1, j} a_{1}(t-j)+\sum_{j=1}^{p} c_{1,2, j} a_{2}(t-j)+\ldots \\
& +\sum_{j=1}^{p} c_{1, S, j} a_{S}(t-j)+e_{1 \mid 1 \ldots S}(t) \\
a_{2}(t)= & \sum_{j=1}^{p} c_{2,1, j} a_{1}(t-j)+\sum_{j=1}^{p} c_{2,2, j} a_{2}(t-j)+\ldots \\
& +\sum_{j=1}^{p} c_{2, S, j} a_{S}(t-j)+e_{2 \mid 1 \ldots S}(t) \\
& \ldots \\
& \ldots \\
a_{S}(t)= & \sum_{j=1}^{p} c_{S, 1, j} a_{1}(t-j)+\sum_{j=1}^{p} c_{S, 2, j} a_{2}(t-j)+\ldots \\
& +\sum_{j=1}^{p} c_{S, S, j} a_{S}(t-j)+e_{S \mid 1, \ldots S}(t)
\end{aligned}
$$


In (2) each equation represents a signal $a_{s}(t)$ at time $t$ that is predicted by previous values of itself and all other signals. For example, in (2), coefficients $c_{1,1, j}(j=1 \ldots P)$ quantitatively describe the influence of the activity of $a_{1}(t)$ on itself, coefficients $c_{1,2, j}$ $(j=1 \ldots P)$ quantitatively describe the influence of the activity of $a_{2}(t)$ on $a_{1}(t)$, and coefficients $c_{1, s, j}(j=1 \ldots P)$ quantitatively describe the influence of signal $a_{s}(t)$ on $a_{1}(t)$, etc. Likewise, coefficients $c_{s, 1, j}(j=1 \ldots P)$ describe the quantitative influence of signal $a_{1}(t)$ on signal $a_{s}(t)$.

To maintain stationarity, a brief "snapshot" of the signal was extracted using the short-window approach (Ding et al., 2000). To derive observations for the AR model, the data window was set at 20 data points and was incrementally moved across the $500 \mathrm{~ms}$ data epoch. The epoch length was 150 data points after down-sampling and these 150 data points fit 13120 -point windows. Thus, this yielded 131 observations per trial. Given that about 300 data trials were recorded from each participant, approximately 39,300 (i.e., $300 \times 131$ ) observations were produced for each participant. The signal was normalized with respect to both the individual trial and ensemble amplitude and variation by detrending each trial, normalizing by the trial mean and standard deviation, and then normalizing by the ensemble mean and standard deviation in a point-by-point manner (Ding et al., 2000; Frye and Wu, in press). Stationarity was verified by examining the unit roots using the Dickey-Fuller test. The details of calculating GC from the AR models are provided in Section "Calculations of Granger Causality Using Least-Squares Linear Regression" in Appendix. To insure that the LSLR algorithm performed adequately, we examined diagnostic residual plots, the leverage values and the condition index to eliminate the possibility of an ill-condition design matrix, bias, or systematic autocorrelations.

The optimal model order is typically chosen by estimating several AR model with different orders and determining which model order optimizes two standard information criteria, the Akaike information criterion (AIC) and the Bayesian information criterion (BIC). Since there is no specific criterion to guide the choice of model orders to test, we selected a wide range of model orders, including 8,12 , and 16 . AIC and BIC were optimized with an order of 16 for all models.

Using the approach above, we constructed a matrix of GC values to represent the influence of each MEG sensor on every other MEG sensor. We then evaluated the significance of each GC value in order to consider only the connections which represented significant connectivity. The same measure of error that is used to calculate GC can also be used in a partial F-test in order to calculate the significance of the GC value. Equation 3 outlines the calculation of this $F$-distributed value which has $P$ and $O \times T-S \times P-1$ degrees of freedom in the numerator and denominator, respectively. Granger used the same symptom " $F$ " to signify GC, making the notation confusing.

$$
F_{P, O \times T-S \times P-1}^{2 \rightarrow s}=\frac{\frac{O \cdot\left(\mathrm{MSE}_{\mathrm{S} \mid 1,3 \ldots S}-\mathrm{MSE}_{\mathrm{s} \mid 1 \ldots S}\right)}{P}}{\frac{\mathrm{O} \cdot \mathrm{MSE}_{\mathrm{s} \mid 1 \ldots S}}{O \cdot T-S \cdot P-1}}
$$

Granger causality values with a corresponding $F$-value that was significant to $p \leq 10^{-4}$ were used further. The average GC values between language areas were calculated by averaging the significant GC values between language areas. This whole process was performed for each frequency band separately.

\section{STATISTICAL ANALYSIS}

In order to quantitatively analyze GC values, we constructed a linear mixed-model similar to models in our recent studies (Frye et al., 2007, 2008, 2009, 2010a,b). In our previous studies we investigated the relation between performance and anatomic connectivity. In these studies we found that this relation was not necessarily the same for both reading groups (i.e., DRs, TRs). Thus, our previous models contained the fixed effects of reading group (TRs vs. DRs), a covariate for performance, and the interaction between these effects. In the current study we examine effective connectivity to/from the IFA. Since there are two directions of connectivity for each connection (in vs. out) an additional fixed effect of connectivity direction is included in the models. Since the IFA is connected to five other areas (i.e., right and left TPA, right and left VOTA, and the contralateral IFA) an additional fixed effect which represented brain area was included in the model. Thus, the final model for this study had fixed effects of area (five levels), reading group (TRs vs. DRs), and connectivity direction (in vs. out) with a covariate representing performance, which in this case is $d$-prime (i.e., sensitivity). The "mixed" procedure of SAS 9.1 (SAS Institute Inc., Cary, NC, USA; see Section "Linear Mixed-Model Used for Statistical Analysis" in Appendix) was used to evaluation the model. Participant, cortical area and connectivity direction were entered as random effects in the mixed-model.

This model provides the ability to test the specific hypothesis of this study. Specifically, it was hypothesized that DRs would have greater top-down connectivity from the left IFA (i.e., outward) to the left hemisphere language areas (TPA, VOTA) as compared to TRs, with this connectivity proportional to performance on the non-word rhyme task. Thus, a four-way interaction was predicted, specifically, a reading group by connectivity direction by performance by area interaction, such that the DRs, but not the TRs, would manifest a relation between performance and outward connectivity from the IFA to the left VOTA and left TPA but not to their right sided homologs.

Each left and right IFA and frequency sub-band was analyzed with a separate mixed-model. For each analysis, all effects along with their interactions were examined for significance. In order to mitigate the effects of inflated alpha due to performing multiple statistical models, we corrected the alpha for the full model using the Bonferroni method. Since there are six frequency bands examined and two IFAs (i.e., left and right) we use an alpha of 0.05/12 =0.004 for the overall analysis. All follow-up statistical tests used an alpha of 0.05 . Model interactions were analyzed by first breaking down the model by reading group and then breaking down the model by connectivity direction. Differences in connectivity between areas were analyzed using orthogonal contrasts. The relationship between performance and connectivity was additionally analyzed using twotailed Pearson correlations. 


\section{RESULTS \\ BETA FREQUENCY SUB-BANDS \\ Low beta (12-14 Hz)}

Left IFA. Left IFA connectivity was influenced by a three-way interaction (i.e., performance by connectivity direction by reading group) $[F(1,52)=15.30, p<0.001]$. To investigate the three-way interaction in more detail each reading group was analyzed separately. The DRs, but not the TRs, were found to demonstrate a performance by connectivity direction interaction $[F(1,56)=57.63$, $p<0.0001]$. Figure 1A depicts the correlation between performance and the difference between inward and outward connectivity. Figure 1A demonstrates that better performance was associated with greater outward as compared to inward connectivity from the left IFA to other brain areas for DRs $(r=-0.96, p<0.01)$. The relationship between performance and difference between inward and outward connectivity was not significant for TRs $(r=0.54$, $p>0.05)$.

To examine this performance by connectivity direction in more detail for the DRs, the relation between performance and connectivity was examined for each connectivity direction separately for DRs. Outward, but not inward, connectivity was associated with performance for DRs $[F(1,28)=8.82, p<0.01]$ such that greater outward connectivity from the left IFA to the other cortical areas (right and left TPA, right and left VOTA, right IFA) was associated with better performance. Figure 1B demonstrates that the correlation between outward connectivity and performance for DRs was significant $(r=0.80, p<0.05)$. The correlation between inward connectivity and performance for DRs was not significant $(r=-0.34, p>0.05)$. These findings suggest that the relationship between the balance in inward and outward connectivity and performance was primarily driven by outward connectivity from the left IFA to other brain regions.

Right IFA. Connectivity between the right IFA and the other cortical areas differed depending on the cortical area $[F(4,60)=5.62$, $p<0.001]$. Planned post hoc contrast demonstrated that across reading groups connectivity between the right IFA and the right TPA was higher than connectivity between the right IFA and the other cortical areas $[t(60)=4.62, p<0.0001$; Figure 1C].

\section{Middle beta (15-19 Hz) and high beta (20-29 Hz)}

Connectivity of the left IFA or right IFA with the other brain areas was not influenced by performance, connectivity direction, cortical area or reading group for the middle or high beta sub-bands.

\section{GAMMA FREQUENCY SUB-BANDS Low gamma $(30-59 \mathrm{~Hz})$}

Connectivity between the left IFA and the other cortical areas differed depending on the cortical areas $[F(4,60)=4.47, p<0.004]$. Planned post hoc contrasts demonstrated that connectivity between the left IFA and the left TPA $[t(60)=2.85, p<0.01]$ and the right TPA $[t(60)=3.45$, $p=0.001]$ was higher than connectivity between the left IFA and the other cortical areas, and connectivity between the left IFA and the left VOTA $[t(60)=3.26, p<0.002]$, right IFA $[t(60)=3.24, p<0.002]$, and the right VOTA $[t(60)=2.63, p=0.01]$ was lower than connectivity between the left IFA and other cortical regions (Figure 2A).

Connectivity between the right IFA and the other brain areas was not influenced by performance, connectivity direction, cortical area, or reading group.

\section{Medium gamma $(60-89 \mathrm{~Hz})$}

Connectivity between the left IFA and the other brain areas was not influenced by performance, connectivity direction, cortical area, or reading group.

Connectivity between the right IFA and the other cortical areas differed depending on the cortical area $[F(4,60)=5.32, p=0.001]$. Planned post hoc contrasts demonstrated that connectivity between the right IFA and the left TPA $[t(60)=3.07, p=0.003]$ and the right TPA $[t(60)=2.77, p<0.01]$ was higher than connectivity between the right IFA and the other cortical areas, and connectivity between the right IFA and the left IFA $[t(60)=2.46, p=0.02]$, left VOTA $[t(60)=3.42, p=0.001]$, and the right VOTA $[t(60)=2.76$, $p<0.01$ ] was lower than connectivity between the right IFA and other cortical areas (Figure 2B).

\section{High gamma $(\mathbf{9 0}-120 \mathrm{~Hz})$}

The relationship between connectivity between the left IFA and the other cortical areas was related to performance but this relationship differed by cortical area (i.e., a performance by cortical
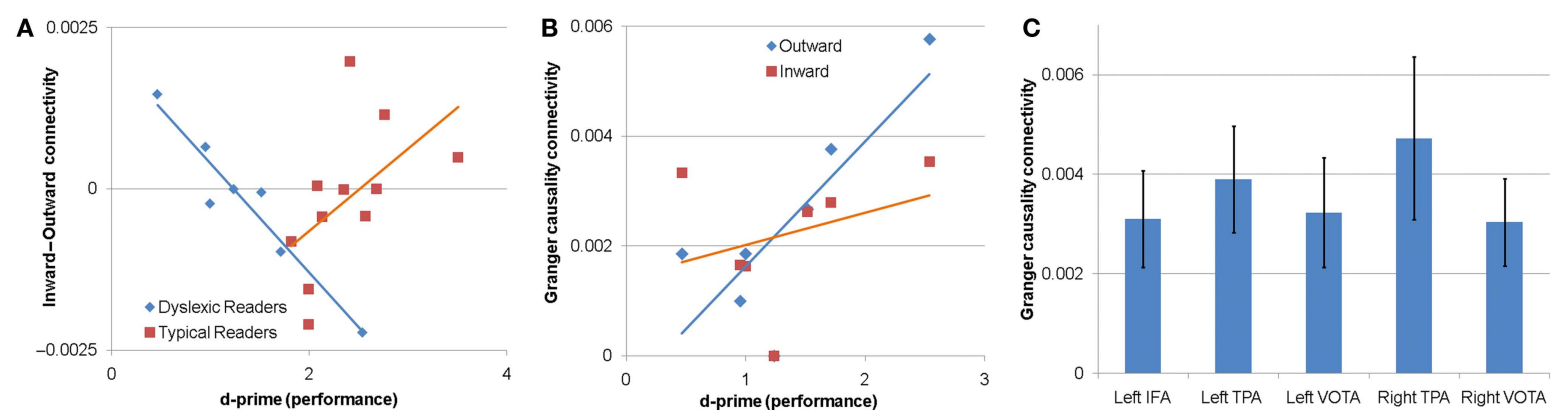

FIGURE 1 | Granger causality connectivity for the low beta sub-band. (A,B) The relationship between performance on the non-word phonological decoding task and Granger causality connectivity for the left inferior frontal area (IFA). (A) The relationship between performance and the difference between inward and outward connectivity. The relationship was only significant for DRs, for which greater outward connectivity (as compared to inward connectivity) from the IFA to other areas was associated with better non-word rhyme discrimination performance (d-prime). (B) The relationship between performance and inward and outward connectivity individually for DRs. Greater outward connectivity from the IFA to other areas was associated with better performance in DRs. (C) Connectivity between the right IFA and the other cortical areas investigated. TPA, temporoparietal area; VOTA, ventral occipital-temporal areas. 


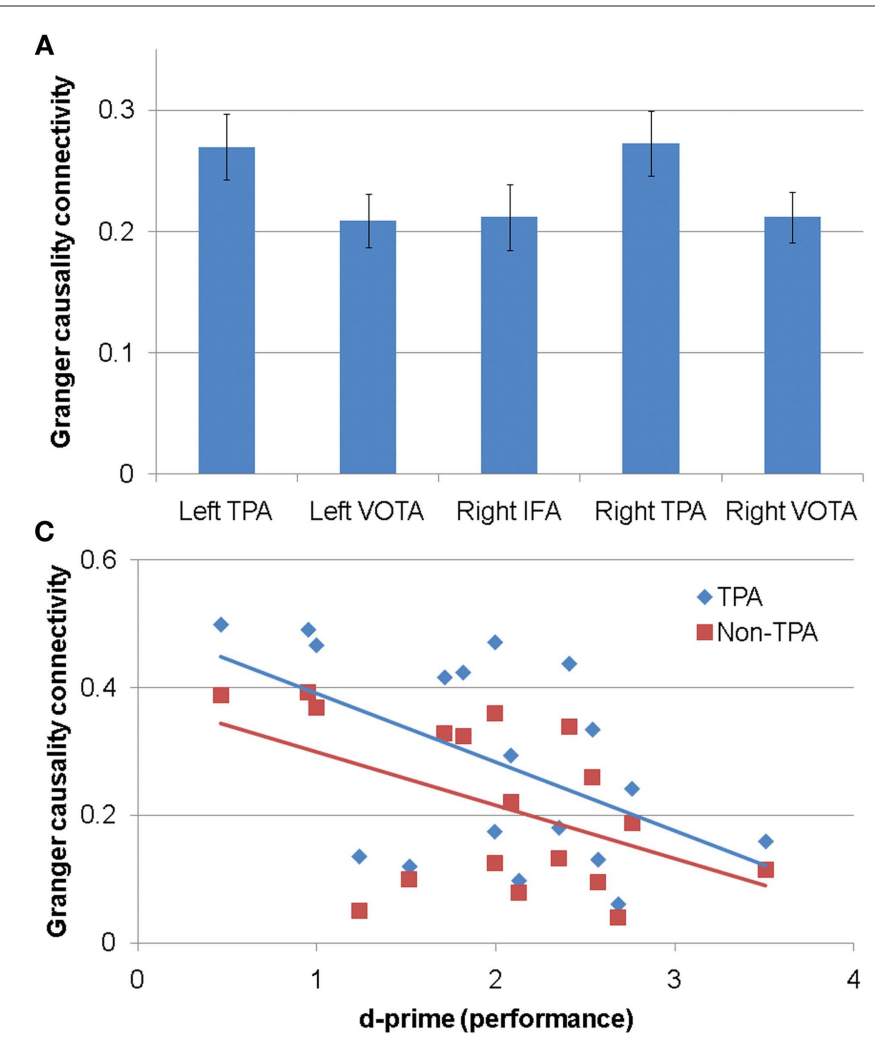

FIGURE 2 | Connectivity for gamma frequency sub-bands. (A) Connectivity between the left inferior frontal area (IFA) and other cortical areas in the low gamma sub-band. (B) Connectivity between the right IFA and other cortical area in the medium gamma sub-band. (C) Relationship between performance and
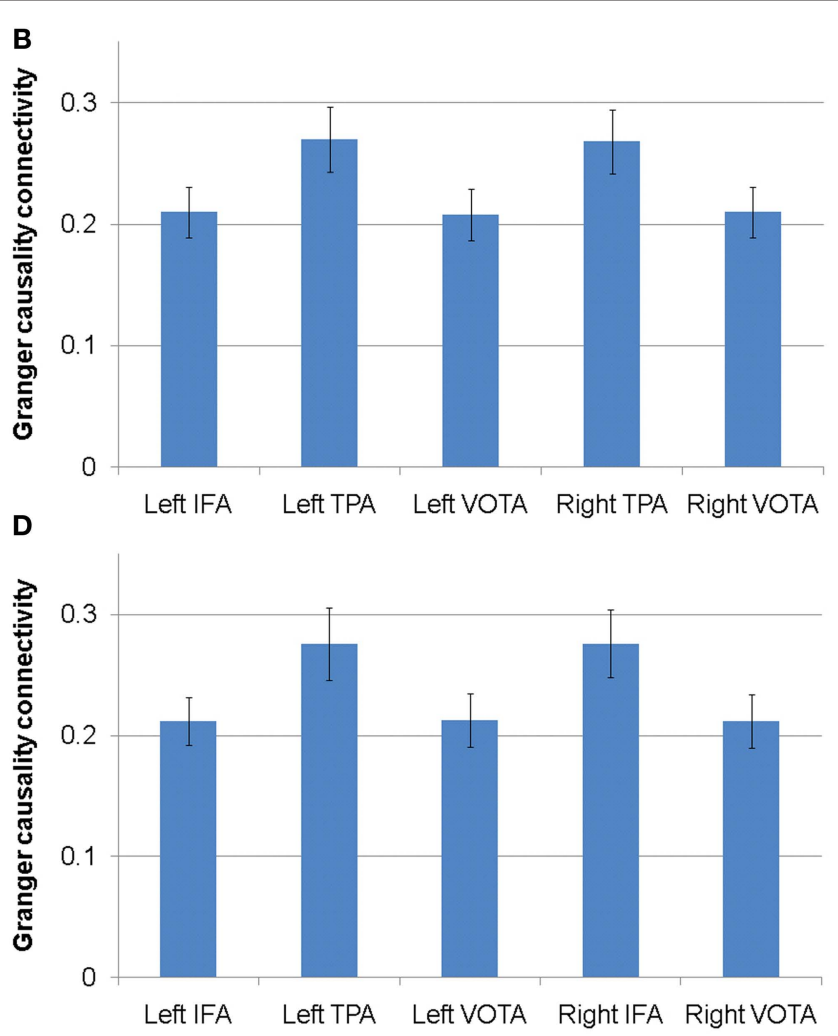

connectivity between the left IFA and temporoparietal (TPA) and nontemporoparietal (non-TPA) areas for the high gamma sub-band. (D) Connectivity between the right IFA and other cortical areas for the high gamma sub-band. VOTA, ventral occipital-temporal areas.

\section{DISCUSSION}

This is the first study to compare effective neuromagnetic connectivity between DRs and TRs and one of the first studies to examine effective connectivity during the pre-stimulus period. In this study, we compared IFA connectivity to and from other brain areas known to be essential for reading (left and right IFA, TPA, and VOTA) in low, medium, and high beta and low, medium, and high gamma frequency sub-bands. One aspect of our hypothesis was that greater top-down connectivity from the left IFA to other cortical regions might serve in a compensatory manner in DRs to facilitate phonological decoding. This hypothesis was confirmed within the low beta sub-band. As predicted, this effect was observed in DRs but not TRs. In Section "Greater Connectivity from the Left IFA to Other Cortical Regions is Associated with Better phonological decoding performance in DRs, but not TRs: Does this reflect a compensatory mechanism in DRs?" we will examine the implications of this finding. In Section "Greater Top-Down Connectivity from the left IFA is not Restricted to Left Hemisphere Reading-Related Structures but Instead Includes Their Right Hemisphere homologs," we will discuss the other aspect of our hypothesis wherein it was predicted that the relationship between IFA connectivity and improved performance would be limited to connections between IFA and left-sided structures used by TRs during phonological decoding. This aspect of the hypothesis was not confirmed since greater left IFA connectivity between both left and right sided homologous structures was 
associated with better phonological decoding performance in DRs. We will discuss the implications of increased bilateral influence of the IFA upon posterior cortical areas. In Section "Greater Gamma Band Effective Connectivity Between Left IFA and Other Regions was Associated with Worse Phonological Decoding Performance in Both Reading Groups" we will discuss our finding that greater effective connectivity in the gamma sub-band was associated with worse phonological decoding. In Section "Different Roles for Beta and the Gamma Preparatory Activity," we will comment on why greater beta connectivity from IFA to other cortical regions was associated with better performance and greater gamma connectivity from IFA to other regions was associated with worse performance. In Section "Future Studies to Examine the Generalizability of These Findings," we will discuss ways to test the generalizability of our findings.

\section{GREATER CONNECTIVITY FROMTHE LEFT IFA TO OTHER CORTICAL REGIONS IS ASSOCIATED WITH BETTER PHONOLOGICAL DECODING PERFORMANCE IN DRs, BUT NOT TRs: DOES THIS REFLECT A COMPENSATORY MECHANISM IN DRs?}

During the pre-stimulus period, within the low beta sub-band, greater top-down effective connectivity from the left IFA to bilateral cortical areas was associated with better phonological decoding performance in DRs, but not TRs. The fact that the changes in effective connectivity were limited to DRs suggests that they might be associated with brain reorganization related to the development of dyslexia as a child. This would be consistent with fMRI studies that have reported over-activation of the left IFA (Hoeft et al., 2007; MacSweeney et al., 2009) in DRs. The fact that outward connectivity from the IFA was associated with performance suggests that such connectivity could be associated with compensatory reorganization. This is consistent with fMRI studies that have shown that left IFA activity increases following remediation (Richards et al., 2002; Temple et al., 2003; Eden et al., 2004). The relationship between the left IFA and improved phonological performance in DRs is also consistent with anatomic connectivity studies. For example, recent diffusion tensor imaging (DTI) studies have demonstrated that phonological performance is related to microstructure of the superior longitudinal fasciculus in DRs (Frye et al., 2010b).

A possible developmental sequence could explain the role that the left IFA has in compensation for the phonological deficit in DRs. Over-activation of the left IFA in preparation for a reading task or during a reading task could represent increased top-down activation of key cortical regions involved in neural language networks for word processing. Remediation training could reinforce the individual's ability to activate language networks in the context of tasks that require word processing. Over time, repeated top-down activation of the posterior language regions by the left IFA could cumulatively strengthen the connectivity between the left IFA and the posterior language areas during development. This is compatible with a recent DTI study that has shown that training therapies for reading change white matter microstructure of pathways in the reading circuit (Keller and Just, 2009). Thus cumulative use of the increased top-down left IFA influence could change white matter microstructure in the pathways connecting the left IFA and posterior language areas and would be compatible with the hypothesis and results of the current study. Of course, a longitudinal study would be needed to test this hypothesized developmental sequence.

\section{GREATER TOP-DOWN CONNECTIVITY FROMTHE LEFT IFA IS NOT RESTRICTED TO LEFT HEMISPHERE READING-RELATED STRUCTURES BUT INSTEAD INCLUDES THEIR RIGHT HEMISPHERE HOMOLOGS}

Although others have recently demonstrated differences in topdown activation from the IFA to other brain regions between DRs and TRs (Cao et al., 2008) this study expands these findings by demonstrating that top-down modulatory activity can be related to phonological task performance on a continuum in DRs. In the current experiment, it was hypothesized that, in DRs, performance would be related to top-down connectivity from the left IFA to the other left-sided posterior language areas typically involved in word processing in TRs. Instead, the performance-related topdown connectivity from the left IFA influenced not only the left, but also the right-sided homologs of the cortical areas typically involved in word processing.

Our results are consistent with the majority of functional imaging studies on DRs which have demonstrated increased right hemisphere activation in DRs as compared to TRs (Simos et al., 2000; Temple, 2002; Heim and Keil, 2004). It is also consistent with studies which have reported increased activation of both left and right hemisphere TPA areas after remediation (Shaywitz et al., 2003; Temple et al., 2003). This pattern of connectivity may explain why many individuals with a history of dyslexia require so many years to develop adequate phonological decoding skills and often continue to have residual problems with reading-related skills well into adulthood. For example, activation of the left TPA and left VOTA by the left IFA in DRs could represent a positive compensatory strategy to activate appropriate posterior language networks. However, concurrent activation of the homologous right hemispheric regions could be somewhat counterproductive and inhibit the occurrence of the necessary neuroplastic changes for specific activation of left hemisphere language networks by leading to the formation of atypical neural networks for reading. Developmental of such atypical language networks could result an extended periods of time (i.e., decades vs. years) for accurate reading skills to develop. Clearly hemispheric asymmetries are important in the development of phonological function, even in individuals without a history of dyslexia. For example, the findings of a recent paper suggest that an intrinsic individual difference in the degree of asymmetry of microstructure of the arcuate fasciculus, a key cortico-cortical pathway involved in connection of anterior and posterior language regions, may predict phonological ability (Lebel and Beaulieu, 2009). Since these asymmetries were found to be age-invariant, these findings could suggest that part of the basis for over-involvement of the right hemispheric decoding network and individual variation in DRs may have to do with the wiring of the arcuate fasciculus from early in life.

\section{GREATER GAMMA BAND EFFECTIVE CONNECTIVITY BETWEEN LEFT IFA AND OTHER REGIONS WAS ASSOCIATED WITH WORSE PHONOLOGICAL DECODING PERFORMANCE IN BOTH READING GROUPS}

This study found that greater gamma band effective connectivity between left IFA and other regions was associated with worse nonword rhyme task performance as measured by $d$-prime within the high gamma sub-band. This relationship was steeper for the left and right TPAs as compared to the other brain areas (i.e., right and left VOTA, right IFA). Therefore during the pre-stimulus period a relatively reduced degree of gamma sub-band effective connectivity between left 
IFA and other areas may be optimal. This is consistent with intracranial EEG data which shown transient desynchronization of gamma activity during a reading task in the left IFA (Lachaux et al., 2008).

\section{DIFFERENT ROLES FOR BETA AND THE GAMMA PREPARATORY ACTIVITY}

The association between better phonological decoding performance and increased outward connectivity between the left IFA and other brain regions was present in the beta (as opposed to the gamma) frequency band. This was predicted because beta activity is thought to operate on an inter-regional spatial scale (Gross et al., 2004, 2006). This may be an example of an advantage of MEG compared to fMRI in terms of GC analysis. Using MEG we were able to break down the connectivity analyses into different frequency sub-bands and the differential effects of increased connectivity in these sub-bands confirms the importance of analyzing the data within narrow frequency bands.

In contrast to beta band activity, gamma band synchronization has been proposed as a mechanism for facilitating communication between neighboring neurons participating in the formation of transient neural networks (Fries, 2007). In fact, transient desynchronization of gamma activity has been shown to occur during a reading task by means of intracranial EEG in the left IFA (Lachaux et al., 2008). Therefore, one interpretation of our findings is that within this high gamma sub-band, strong coupling between the nodes of the neural network during the pre-stimulus period may reduce the ability of the network to decouple and reorganize into large-scale cognitive networks during the performance of the actual task. In particular, tighter intercortical connectivity between the two TPAs during the pre-stimulus period may interfere with a necessary shift toward left TPA ascendency. We suggest that this may result in slower and less automatic pseudoword phonological decoding.

\section{FUTURE STUDIES TO EXAMINE THE GENERALIZABILITY OF THESE FINDINGS}

This study examines connectivity between language regions during the pre-stimulus period of a non-word phonological decoding task. Other word stimuli such as regular and irregular words may be processed through different neural networks as compared to non-words. It is not known whether the patterns of cortical connectivity identified in this study will also occur during the prestimulus period of tasks requiring processing of other word types. In fact, it is very possible that the patterns of cortical connectivity identified may not be word or language specific at all. For example, we expected top-down activity from the IFA to have a significant influence on the posterior language networks that subserve reading which are left hemisphere lateralized. What we found is that the IFA appeared to have equal top-down influence on all brain regions examined, suggesting that this influence is not word specific. In future studies, the pre-stimulus period associated with a non-word task should be compared to the pre-stimulus period associated with tasks requiring the processing of other types of word features

\section{REFERENCES}

Ackerman, P. T., Dykman, R. A., Oglesby, D. M., and Newton, J. E. (1994). EEG power spectra of children with dyslexia, slow learners, and normally reading children with add during verbal processing. J. Learn. Disabil. 27, 619-630.

Aron, A. R., and Poldrack, R. A. (2005). The cognitive neuroscience of response inhibition: relevance for genetic research in attention-deficit/

including tasks focusing on orthographic and semantic word properties and stimuli other than words. Such studies will provide an understanding of whether the pattern of pre-stimulus connectivity found in this study is specific to phonological non-word decoding, word decoding or represents a more general pattern of pre-stimulus preparatory connectivity. Future studies should also incorporate additional participants, to afford the possibility of examining differences in brain connectivity attributable to gender.

\section{CONCLUSION}

This study has demonstrated that pre-stimulus preparatory networks are reorganized in DRs and that network structure is directly associated with non-word rhyme performance. Reorganization of the pre-stimulus network associated with DRs was found in the beta frequency band. Although preliminary, this finding of a performance-related relationship with IFA effective connectivity sheds light on previous functional neuroimaging findings of over-activation of the left IFA in DRs as compared to TRs. Over-activation of the left IFA in DRs during reading tasks may in fact reflect increased topdown influence of the left IFA. The relationship of IFA function to more general cognitive abnormalities associated with dyslexia is not clear, but given the number of studies demonstrating subtle deficits in executive function in DRs (Narhi et al., 1997; Lazar and Frank, 1998; Willcutt et al., 2001, 2005; Brosnan et al., 2002; Gioia et al., 2002), further investigation of the IFA in DRs may be fruitful.

Additionally, across reading groups, phonological decoding performance diminished in proportion to the strength of the interregional gamma connectivity during the pre-stimulus period. Therefore, individual differences in gamma frequency band activity affected rhyme task performance in a continuous manner across both DRs and TRs.

This combination of results demonstrates the importance of considering direction in connectivity analysis and suggests that analyses based on GC can help uncover the typical and atypical architecture of neural networks that underlie cognition. Future studies should attempt to analyze source localized MEG activation rather than sensor localized activation in order to more accurately examine cortical location. In the future, experimental paradigms which combine MEG and fMRI may also help better spatially localize these cognitive networks and help understand the discrepancies in the findings of these two imaging modalities in DRs. This will no doubt provide a more in-depth understanding of the neural networks that subserve reading in DRs. Such an understanding will help guide the development of innovative remediation programs and allow DRs to develop the ability to accurately read at a younger age.

\section{ACKNOWLEDGMENTS}

This study was supported by grant NS046565 to Dr. Richard E. Frye and in part by CELEST, an NSF Science of Learning Center (SBE-0354378), Jacqueline Liederman, Investigator.

hyperactivity disorder. Biol. Psychiatry 57, 1285-1292.

Aylward, E.H., Richards, T.L., Berninger,V. W., Nagy, W. E., Field, K. M., Grimme, A. C., Richards, A. L., Thomson, J. B., and Cramer, S. C. (2003). Instructional treatment associated with changes in brain activation in children with dyslexia. Neurology 61, 212-219.

Bitan, T., Cheon, J., Lu, D., Burman, D. D., and Booth, J.R.(2009). Developmental increase in top-down and bottom-up 
processing in a phonological task: an effective connectivity, fMRI study. J. Cogn. Neurosci. 21, 1135-1145.

Brosnan, M., Demetre, J., Hamill, S., Robson, K., Shepherd, H., and Cody, G. (2002). Executive functioning in adults and children with developmental dyslexia. Neuropsychologia 40, 2144-2155.

Brunswick, N., McCrory, E., Price, C. J., Frith, C. D., and Frith, U. (1999). Explicit and implicit processing of words and pseudowords by adult developmental dyslexics: a search for Wernicke's Wortschatz? Brain 122, 1901-1917.

Cao, F., Bitan, T., and Booth, J. R. (2008). Effective brain connectivity in children with reading difficulties during phonological processing. Brain Lang. 107, 91-101.

Cao, F., Bitan, T., Chou, T. L., Burman, D. D., and Booth, J. R. (2006). Deficient orthographic and phonological representations in children with dyslexia revealed by brain activation patterns. J. Child. Psychol. Psychiatry 47, 1041-1050.

Cohen, D., Schalpfer, U., Ahlfors, S., Hamalainen, M., and Halgren, E. (2002). New six-layer magnetically shielded room for MEG. Biomag Proceed.

Cornelissen, P. L., Kringelbach, M. L., Ellis, A. W., Whitney, C., Holliday, I. E., and Hansen, P.C. (2009). Activation of the left inferior frontal gyrus in the first 200 ms of reading: evidence from magnetoencephalography (MEG). PLOS ONE 4, e5359. doi: 10.1371/ journal.pone.0005359.

Dale, A. M., Fischl, B., and Sereno, M. I. (1999). Cortical surface-based analysis. I. Segmentation and surface reconstruction. Neuroimage 9, 179-194.

Ding, M., Bressler, S. L., Yang, W., and Liang, H. (2000). Short-window spectral analysis of cortical eventrelated potentials by adaptive multivariate autoregressive modeling: data preprocessing, model validation, and variability assessment. Biol. Cybern. $83,35-45$.

Ding, M., Chen, Y., and Bressler, S. L. (2006). "Granger causality: basic theory and application to neuroscience," in Handbook of Time Series Analysis, eds B. Schelter, M. Winterhalder, and J. Timmer (Berlin: Wiley-VCH), 451-474.

Dragovic, M. (2004). Categorization and validation of handedness using latent class analysis. Acta Neuropsychiatr. 16, 212-218.

Duncan Milne, R., Hamm, J. P., Kirk, I. J., and Corballis, M. C. (2003). Anteriorposterior beta asymmetries in dyslexia during lexical decisions. Brain Lang. 84, 309-317.
Eden, G. F., Jones, K. M., Cappell, K., Gareau, L., Wood, F. B., Zeffiro, T. A., Dietz, N. A., Agnew, J.A., and Flowers, D. L. (2004). Neural changes following remediation in adult developmental dyslexia. Neuron 44, 411-422.

Fischl, B., Sereno, M. I., and Dale, A. (1999).Cortical surface-based analysis. II: inflation, flattening, and a surfacebased coordinate system. Neuroimage 9, 195-207.

Fries, P. (2007). A mechanism for cognitive dynamics: neuronal communication through neuronal coherence. Trends Cogn. Sci. 9, 474-480.

Frye, R. E., Fisher, J. M., Coty, A., Zarella, M., Liederman, J., and Halgren, E. (2007). Linear coding of voice onset time. J. Cogn. Neurosci. 19, 1476-1487.

Frye, R. E., Fisher, J.M., Witzel, T., Ahlfors, S. P., Swank, P., Liederman, J., and Halgren, E. (2008). Objective phonological and subjective perceptual characteristics of syllables modulate spatiotemporal patterns of superior temporal gyrus activity. Neuroimage 40, 1888-1901.

Frye, R. E., Hasan, K., Xue, L., Strickland, D., Malmberg, B., Liederman, J., and Papanicolaou, A. C. (2009). Splenium microstructure is related to two dimensions of reading skill. Neuroreport 19 , 1627-1631.

Frye, R. E., Liederman, J., Malmberg, B., McLean, J., Strickland, D., and Beauchamp, M. S. (2010a). Surface area accounts for the relation of gray matter volume to reading-related skills and history of dyslexia. Cereb. Cortex. 20, 2625-2635.

Frye, R. E., Liederman, J., Hasan, K. M., Lincoln, A., Malmberg, B., deSouza, L., McLean, J., and Papanicolaou, A. (2010b). Diffusion tensor quantification of the relations between microstructural and macrostructural indices of white matter and reading. Hum. Brain Mapp. doi: 10.1002/hbm.21103

Frye, R. E., and Wu, M.-H. (in press) Multichannel least-squares linear regression provides a fast, accurate, unbiased and robust estimation of Granger causality for neurophysiological data. Comput. Biol. Med.

Galaburda, A. M., Sherman, G. F., Rosen, G. D., Aboitiz, F., and Geschwind, N. (1985). Developmental dyslexia: four consecutive patients with cortical anomalies. Ann. Neurol. 18, 222-233.

Gioia, G. A., Isquith, P. K., Kenworthy, L., and Barton, R. M. (2002). Profiles of everyday executive function in acquired and developmental disorders. Child Neuropsychol. 8, 121-137.

Granger, C. W. J. (1969). Investigating causal relations by econometric models and cross-spectral methods. Econometrica 37, 424-438.
Gross, J., Schmitz, F., Schnitzler, I., Kessler K., Shapiro, K., Hommel, B., and Schnitzler, A. (2004). Modulation of long-range neural synchrony reflects temporal limitations of visual attention in humans. Proc. Natl. Acad. Sci. U.S.A. 101, 13050-13055.

Gross, J., Schmitz, F., Schnitzler, I., Kessler, K., Shapiro, K., Hommel, B., and Schnitzler, A. (2006). Anticipatory control of long-range phase synchronization. Eur. J. Neurosci. 24, 2057-2060.

Hämäläinen, M., Hari, R., Ilmoniemi, R., Knuutila, J., and Lounasmaa, O. (1993). Magnetoencephalography theory, instrumentation, and applications to noninvasive studies of the working human brain. Rev. Mod. Phys. 65, 1-93.

Hampshire, A., Thompson, R., Duncan, J., and Owen, A. M. (2010). Lateral prefrontal cortex subregions make dissociable contributions during fluid reasoning. Cereb. Cortex. doi: 10.1093/ cercor/bhq085

Heim, S., and Keil, A. (2004). Large-scale neural correlates of developmental dyslexia. Eur. Child Adolesc. Psychiatry 13, 125-140.

Hoeft, F., Hernandez, A., McMillon, G., Taylor-Hill, H., Martindale, J. L., Meyler, A., Keller, T. A., Siok, W. T. Deutsch, G. K., Just, M. A., WhitfieldGabrieli, S., and Gabrieli, J. D. (2006). Neural basis of dyslexia: a comparison between dyslexic and nondyslexic children equated for reading ability. $J$. Neurosci. 26, 10700-10708.

Hoeft, F., Meyler, A., Hernandez, A., Juel, C., Taylor-Hill, H., Martindale, J. L. McMillon, G., Kolchugina, G., Black, J. M., Faizi, A., Deutsch, G. K., Siok, W. T., Reiss, A. L., Whitfield-Gabrieli, S. and Gabrieli, J. D. (2007). Functional and morphometric brain dissociation between dyslexia and reading ability. Proc. Natl. Acad. Sci. U.S.A. 104 4234-4239.

Horwitz, B., Rumsey, J. M., and Donohue, B. C. (1998). Functional connectivity of the angular gyrus in normal reading and dyslexia. Proc. Natl. Acad. Sci. U.S.A. 95, 8939-8944.

Humphreys, P., Kaufmann, W. E. and Galaburda, A. M. (1990). Developmental dyslexia in women: neuropathological findings in three patients. Ann. Neurol. 28, 727-738.

Kaufmann, W. E., and Galaburda, A. M. (1989). Cerebrocortical microdysgenesis in neurologically normal subjects: a histopathologic study. Neurology 39 , 238-244.

Keller, T. A., and Just, M. A. (2009). Altering cortical connectivity. Neuron 64, 624-631.

Kenner, N. M., Mumford, J. A., Hommer, R. E., Skup, M., Leibenluft, E., and
Poldrack, R. A. (2010). Inhibitory motor control in response stopping and response switching. J. Neurosci. 30, 8512-8518.

Klimesch, W., Doppelmayr, M., Wimmer, H., Gruber, W., Rohm, D., Schwaiger, J. and Hutzler, F. (2001). Alpha and beta band power changes in normal and dyslexic children. Clin. Neurophysiol. 112, 1186-1195.

Koyama, M. S., Kelly, C., Shehzad, Z., Penesetti, D., Castellanos, F. X., and Milham, M. P. (2010). Reading networks at rest. Cereb. Cortex 20, 2549-2559.

Lachaux, J.P., Jung, J., Mainy, N., Dreher, J. C., Bertrand, O., Baciu, M., Minotti, L., Hoffmann, D., and Kahane, P. (2008). Silence is golden: transient neural deactivation in the prefrontal cortex during attentive reading. Cereb. Cortex $18,443-450$.

Lazar, W., and Frank, Y. (1998). Frontal systems dysfunction in children with attention-deficit/hyperactivity disorder and learning disabilities. J. Neuropsychiatry Clin. Neurosci. 10, 160-167.

Lebel, C., and Beaulieu, C. (2009). Lateralization of the arcuate fasciculus from childhood to adulthood and its relation to cognitive abilities in children. Hum. Brain Mapp. 30, 3563-3573.

Levy, J., Pernet, C., Treserras, S., Boulanouar, K., Aubry, F., Demonet, J. F., and Celsis, P. (2009). Testing for the dual-route cascade reading model in the brain: an fMRI effective connectivity account of an efficient reading style. PLoS ONE 4, e6675. doi: 10.1371/ journal.pone.0006675.

Liang, H., Bressler, S. L., Ding, M. Truccolo, W. A., and Nakamura, R. (2002). Synchronized activity in prefrontal cortex during anticipation of visuomotor processing. Neuroreport 13, 2011-2015.

MacSweeney, M., Brammer, M. J., Waters, D., and Goswami, U. (2009). Enhanced activation of the left inferior frontal gyrus in deaf and dyslexic adults during rhyming. Brain 132, 1928-1940.

Mainy, N., Jung, J., Baciu, M., Kahane, P., Schoendorff, B., Minotti, L., Hoffmann, D., Bertrand, O., and Lachaux, J. P. (2008). Cortical dynamics of word recognition. Hum. Brain Mapp. 29, 1215-1230.

Maisog, J. M., Einbinder, E. R., Flowers, D. L., Turkeltaub, P. E., and Eden, G. F. (2008). A meta-analysis of functional neuroimaging studies of dyslexia. Ann. N. Y. Acad. Sci. 1145, 237-259.

Matsumoto, A., and Iidaka, T. (2008). Gamma band synchronization and the formation of representations in visual word processing: evidence from repetition and homophone priming. $J$. Cogn. Neurosci. 20, 2088-2096. 
McGraw Fisher, J., Liederman, J., Johnson, J., Lincoln, A., and Frye, R. (in press). A demonstration that task difficulty can confound the interpretation of lateral differences in brain activation between typical and dyslexic readers. Laterality.

Miller-Shaul S. (2005). The characteristics of young and adult dyslexic readers on reading and reading related cognitive tasks as compared to normal readers. Dyslexia 11, 132-151.

Narhi, V., Rasanen, P., Mesapelto, R. L., and Ahonen, T. (1997). Trail making test in assessing children with reading disabilities: a test of executive functions or content information. Percept. Mot. Skills 84, 1355-1362.

Ortiz, T., Exposito, F. J., Miguel, F., MartinLoeches, M., and Rubia, F. J. (1992). Brain mapping in dysphonemic dyslexia: in resting and phonemic discrimination conditions. Brain Lang. 42, 270-285.

Pennington, B. F., and Lefly, D. L. (2001). Early reading development in children at family risk for dyslexia. Child Dev. 72, 816-833.

Penolazzi, B., Spironelli, C., Vio, C., and Angrilli, A. (2010). Brain plasticity in developmental dyslexia after phonological treatment: a beta EEG band study. Behav. Brain Res. 209, 179-182.

Pugh, K. R., Mencl, W. E., Jenner, A. R., Katz, L., Frost, S. J., Lee, J. R., Shaywitz, S. E., and Shaywitz, B. A. (2000a). Functional neuroimaging studies of reading and reading disability (developmental dyslexia).Ment. Retard. Dev. Disabil. Res. Rev. 6, 207-213.

Pugh, K. R., Mencl, W. E., Shaywitz, B. A., Shaywitz, S. E., Fulbright, R. K., Constable, R. T., Skudlarski, P., Marchione, K. E., Jenner, A. R., Fletcher, J. M., Liberman, A. M., Shankweiler, D. P., Katz, L., Lacadie, C., and Gore, J. C. (2000b). The angular gyrus in developmental dyslexia: task-specific differences in functional connectivity within posterior cortex. Psychol. Sci. 11, 51-56.

Quaglino, V., Bourdin, B., Czternasty, G., Vrignaud, P., Fall, S., Meyer, M.
E., Berquin, P., Devauchelle, B., and de Marco, G. (2008). Differences in effective connectivity between dyslexic children and normal readers during a pseudoword reading task: an fMRI study. Neurophysiol. Clin. 38, 73-82.

Richards, T., Berninger, V., Winn, W., Stock, P., Wagner, R., Muse, A., and Maravilla, K. (2007). Functional MRI activation in children with and without dyslexia during pseudoword aural repeat and visual decode: before and after treatment. Neuropsychology 21, 732-741.

Richards, T. L., Berninger, V. W., Aylward, E. H., Richards, A. L., Thomson, J. B., Nagy, W. E., Carlisle, J. F., Dager, S. R., and Abbott, R. D. (2002). Reproducibility of proton MR spectroscopic imaging (PEPSI): comparison of dyslexic and normal-reading children and effects of treatment on brain lactate levels during language tasks. AJNR Am. J. Neuroradiol. 23, 1678-1685.

Richlan, F., Kronbichler, M., and Wimmer, H. (2009). Functional abnormalities in the dyslexic brain: a quantitative metaanalysis of neuroimaging studies. Hum. Brain Mapp. 30, 3299-3308.

Shaywitz, B. A., Skudlarski, P., Holahan, J. M., Marchione, K. E., Constable, R. T., Fulbright, R. K., Zelterman, D., Lacadie, C., and Shaywitz, S. E. (2007). Age-related changes in reading systems of dyslexic children. Ann. Neurol. 61, 363-370.

Shaywitz, S. E. (1998). Dyslexia. N. Engl. J. Med. 338, 307-312.

Shaywitz, S.E., and Shaywitz, B. A. (2005). Dyslexia (specific reading disability). Biol. Psychiatry 57, 1301-1309.

Shaywitz, S. E., and Shaywitz, B. A. (2008). Paying attention to reading: the neurobiology of reading and dyslexia. Dev. Psychopathol. 20, 1329-1349.

Shaywitz, S. E., Shaywitz, B. A., Fulbright, R. K., Skudlarski, P., Mencl, W. E., Constable, R. T., Pugh, K. R., Holahan, J. M., Marchione, K. E., Fletcher, J. M., Lyon, G. R., and Gore, J. C. (2003). Neural systems for compensation and persistence: young adult outcome of childhood reading disability. Biol. Psychiatry 54, 25-33.
Simos, P. G., Breier, J. I., Fletcher, J. M., Bergman, E., and Papanicolaou, A. C. (2000). Cerebral mechanisms involved in word reading in dyslexic children: a magnetic source imaging approach. Cereb. Cortex 10, 809-816.

Simos, P. G., Fletcher, J. M., Denton, C., Sarkari, S., Billingsley-Marshall, R., and Papanicolaou, A. C. (2006). Magnetic source imaging studies of dyslexia intervention. Dev. Neuropsychol. 30, 591-611.

Simos, P. G., Fletcher, J. M., Sarkari, S., Billingsley, R. L., Denton, C., and Papanicolaou, A. C. (2007). Altering the brain circuits for reading through intervention: a magnetic source imaging study. Neuropsychology 21 , 485-496.

Snowling, M. J. (2008). Specific disorders and broader phenotypes: the case of dyslexia.Q. J. Exp. Psychol. (Colchester) 61, 142-156.

Spironelli, C., Penolazzi, B., and Angrilli, A. (2008). Dysfunctional hemispheric asymmetry of theta and beta EEG activity during linguistic tasks in developmental dyslexia. Biol. Psychol. 77, 123-131.

Svensson, I., and Jacobson, C. (2006). How persistent are phonological difficulties? A longitudinal study of reading retarded children. Dyslexia 12, 3-20.

Tamnes, C. K., Østby, Y., Walhovd, K. B. Westlye, L. T., Due-Tønnessen, P., and Fjell, A. M. (2010). Neuroanatomical correlates of executive functions in children and adolescents: a magnetic resonance imaging (MRI) study of cortical thickness. Neuropsychologia 48, 2496-2508.

Temple, E. (2002). Brain mechanisms in normal and dyslexic readers. Curr. Opin. Neurobiol. 12, 178-183.

Temple, E., Deutsch, G. K., Poldrack, R.A., Miller, S. L., Tallal, P., Merzenich, M. M., and Gabrieli, J. D. (2003). Neural deficits in children with dyslexia ameliorated by behavioral remediation: evidence from functional MRI. Proc. Natl. Acad. Sci. U.S.A. 100, 2860-2865.

Trebuchon-Da Fonseca, A., Benar, C. G. Bartolomei, F., Regis, J., Demonet, J. F., Chauvel, P., and Liegeois-Chauvel,
C. (2009). Electrophysiological study of the basal temporal language area: a convergence zone between language perception and production networks. Clin. Neurophysiol. 120, 539-550.

von Stein, A., Rappelsberger, P., Sarnthein, J., and Petsche, H. (1999). Synchronization between temporal and parietal cortex during multimodal object processing in man. Cereb. Cortex 9, 137-150

Wechsler, D. (1997). Wechsler Adult Intelligence Scale-III (WAIS-III). New York: Psychological Corporation.

Willcutt, E. G., Pennington, B. F., Boada, R., Ogline, J.S., Tunick, R. A., Chhabildas, N. A., and Olson, R. K. (2001). A comparison of the cognitive deficits in reading disability and attentiondeficit/hyperactivity disorder. $J$. Abnorm. Psychol. 110, 157-172.

Willcutt, E. G., Pennington, B. F., Olson, R. K., Chhabildas, N., and Hulslander, J. (2005). Neuropsychological analyses of comorbidity between reading disability and attention deficit hyperactivity disorder: in search of the common deficit. Dev. Neuropsychol. 27, 35-78.

Conflict of Interest Statement: The authors declare that the research was conducted in the absence of any commercial or financial relationships that could be construed as a potential conflict of interest.

Received: 15 June 2010; accepted: 08 November 2010; published online: 02 December 2010

Citation: Frye RE, Wu M-H, Liederman J and McGraw Fisher J (2010) Greater prestimulus effective connectivity from the left inferior frontal area to other areas is associated with better phonological decoding in dyslexic readers. Front. Syst. Neurosci. 4:156. doi: 10.3389/fnsys.2010.00156 Copyright (c) 2010 Frye, Wu, Liederman and McGraw Fisher. This is an open-access article subject to an exclusive license agreement between the authors and the Frontiers Research Foundation, which permits unrestricted use, distribution, and reproduction in any medium, provided the original authors and source are credited. 


\section{APPENDIX \\ CALCULATIONS OF GRANGER CAUSALITY USING LEAST-SQUARES LINEAR REGRESSION}

In order to formulate this problem for LSLR we define matrix $\mathrm{X}^{\mathrm{o}}$ for one data observation o as (A.1). The design matrix (A.2) is then defined for all observations from (A.1). The dependent matrix (A.3) is then defined from a series of O observations for each signal $s$. The coefficients for the above set of equations can then be solved for each signal s using the $\mathrm{X}$ and $\mathrm{Y}_{\mathrm{s}}$ (A.4). The coefficients derived with (A.4) are the same coefficients outlined in Eq. 2. For each signal $s$, Eq. A.4 derives a coefficient matrix with coefficients $\left[c_{s, 1,1} \ldots c_{s, 1, P} \ldots \ldots c_{s, S, 1} \ldots c_{s, S, P}\right]$. Using the coefficients, the error of the AR for each source can be calculated using (A.5). The variance of the model error, also known as the mean squared error (MSE) for signal $s$ is shown in Eq. A.6.

$$
\begin{aligned}
& \mathrm{X}^{\mathrm{o}}=\left[a_{1}^{\mathrm{o}}(t-1) \ldots a_{1}^{\mathrm{o}}(t-P) a_{2}^{\mathrm{o}}(t-1) \ldots a_{2}^{\mathrm{o}}(t-P) \ldots\right. \\
& \left.a_{S}^{\mathrm{o}}(t-1) \ldots a_{S}^{\mathrm{o}}(t-P)\right] \\
& \mathrm{X}=\left[\mathrm{X}^{1} \ldots \mathrm{X}^{\mathrm{o}}\right]^{\prime} \\
& \mathrm{Y}_{s}=\left[\mathrm{a}_{\mathrm{s}}^{1}(t) \ldots \mathrm{a}_{s}^{\mathrm{o}}(t)\right] \\
& C_{s}=\left(X^{\prime} X\right)^{-1}\left(X^{\prime} Y_{s}\right) \\
& \mathrm{e}_{s \mid 1 \ldots s}=X c_{s}-Y_{s} \\
& \operatorname{MSE}_{s \mid 1 \ldots S}=\frac{\sum_{i=1}^{O} \mathrm{e}_{s \mid 1 . \ldots S}^{2}}{O}
\end{aligned}
$$

Granger causality is a measure of the influence of one signal on another signal. This measure is based on the relative change in the model error when an independent signal is added to the AR model to improve the prediction of the dependent signal (Granger, 1969). For example, the signal $A=[a(t): 1 \ldots T]$ can be predicted by itself using as AR model as given in (A.7).

$a(t)=\sum_{j=1}^{p} c_{a, j} \times a(t-j)+\mathrm{e}_{a \mid a}(t)$

In a similar manner, signal $A$ can also be predicted by signal $B=[b(t): 1 \ldots T]$ as represented by (A.8).

$a(t)=\sum_{j=1}^{p} c_{b, j} \times b(t-j)+\mathrm{e}_{a \mid b}(t)$

Similar to the equations provided in (2), signal $A$ can also be predicted by itself, $A$, and another signal, $B$, as presented in (A.9).

$a(t)=\sum_{j=1}^{p} c_{a, j} \times a(t-j)+\sum_{k=1}^{p} c_{b, j} \times b(t-k)+\mathrm{e}_{a \mid a b}(t)$

Granger causality (Ding et al., 2006) is calculated as the ratio of the variance of the model error before and after the addition of a new signal. We can calculate the GC of signal $B$ on signal $A$ using (A.10).
$F_{b \rightarrow a}=\ln \frac{\operatorname{Var}\left(\mathrm{e}_{a \mid a}\right)}{\operatorname{Var}\left(\mathrm{e}_{a \mid a b}\right)}$

We can apply the same calculation to the system of AR models presented in (2). The AR models for a signal $s$ in (2) already accounts for the influence of all of signals, similar to (A.9) in the example above. We can now eliminate the signal of interest by reconstructing the matrix $\mathrm{X}^{\circ}$ leaving out the signal of interest. For example, if we were interested in the influence of signal 2 on any other signal $s$, we would reformulate $\mathrm{X}^{\circ}$ as demonstrated in (A.11), recalculate the LSLR and derive the error vector $e_{s \mid 1,3 \ldots s^{*}}$ Granger causality of the influence of signal 2 on signal s given all of the other signals 1 to $S$ (expect for 2) would be calculated with (A.12).

$$
\begin{aligned}
\mathrm{X}^{\mathrm{o}}=[ & a_{1}^{\mathrm{o}}(t-1) \ldots a_{1}^{\mathrm{o}}(t-P) a_{3}^{\mathrm{o}}(t-1) \ldots a_{3}^{\mathrm{o}}(t-P) \\
& \left.\ldots a_{S}^{\mathrm{o}}(t-1) \ldots a_{S}^{\mathrm{o}}(t-P)\right] \\
F_{2 \rightarrow s}= & \ln \frac{\mathrm{MSE}_{\mathrm{S} \mid 1,3 \mathrm{~S}}}{\mathrm{MSE}_{\mathrm{S} \mid 1 \ldots \mathrm{S}}}
\end{aligned}
$$

\section{LINEAR MIXED-MODEL USED FOR STATISTICAL ANALYSIS}

The general mixed-model is in the matrix form:

$y=X \beta+Z \gamma+\varepsilon$

where $y$ is the dependent variable, which in this case is connectivity between two cortical regions, $\mathrm{X}$ is the design matrix for the fixed effects and covariate, $\beta$ is a vector containing the parameters of the fixed effects and covariate, $\mathrm{Z}$ is the design matrix for the random effects, $\gamma$ contains the parameters of the random effects and $\varepsilon$ is the variance-covariance matrix of the model error. The key assumption of the mixed model are that both $\gamma$ and $\varepsilon$ have the expected value of 0 (i.e., $E(\gamma)=0$ and $E(\varepsilon)=0$ ) and known covariance structure given by the matrixes $\operatorname{Var}(\gamma)$ and $\operatorname{Var}(\varepsilon)$. The values for each row of the design matrix $\mathrm{X}$ are given by:

$$
\begin{aligned}
& c \text { area }_{1} \text { area }_{2} \text { area }_{3} \text { area }_{4} \text { area }_{5} \text { inout read } \\
& d_{\mathrm{p}}\left(\text { area }_{1} \times \text { inout } \ldots\left(\text { area }_{5} \times \text { inout }\right)\right. \\
& \left(\text { area }_{1} \times \text { read }\right) \ldots\left(\text { area }_{5} \times \text { read }\right) \ldots\left(\text { area }_{1} \times d_{\mathrm{p}}\right) \ldots \\
& \left(\text { area }_{5} \times d_{\mathrm{p}}\right)(\text { inout } \times \text { read })
\end{aligned}
$$

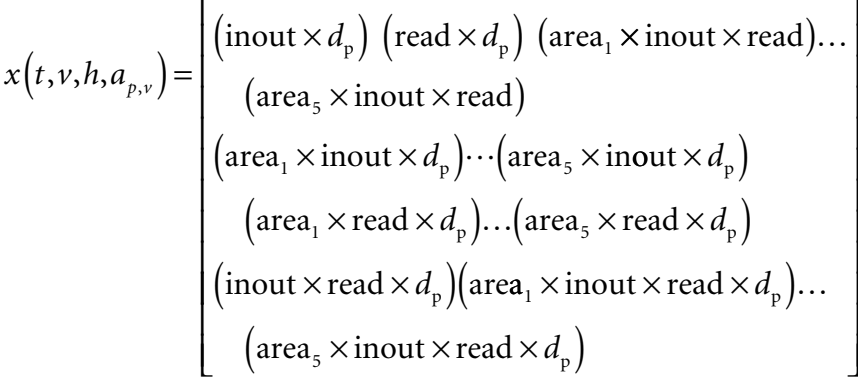

where $c$ is the constant with value 1 , area is the cortical area represented by the five dummy variables $\operatorname{area}_{1} \ldots$ area $_{5}$ (i.e., for analysis of the left frontal region, dummy variables would be set up to represent the other cortical regions that the left frontal region is connected 
to, for example area ${ }_{1}=1$ for left TPA and 0 otherwise, area $_{2}=1$ for left occipital and 0 otherwise, etc.), inout is connectivity direction represented by a dummy variable (i.e., inward $=0$, outward $=1$ ), read is reading group as represented by a dummy variable (i.e., dyslexia $=1$, typical $=0$ ) and $d_{\mathrm{p}}$ is the centered $d$-prime value for the particular participant, $p$. The values for each row of the randomeffects design matrix $Z$ are given by $z(p$, area,inout $)$
$\quad=\left[p_{1} \ldots p_{17}\right.$ area area $_{2}$ area $_{3}$ area $_{4}$ area $_{5}$ inout $]$

where $p$ is the participant where $p_{i}$ is 1 for participant $i$ and 0 otherwise. The mixed-model was calculated using the restricted maximum likelihood method. 